\title{
Anchoring: Cognitive bias and numerical conception
}

\author{
PÀVlos KipOURÀS \\ ORCID: 0000-0002-4021-7584 \\ Scuola di Grafopatologia Forense (SGF), Naples, Italy \\ Department of Forensic Science, Criminal Procedure and Judicial Expertise \\ Institute of Law, South Ural State University, Russia
}

\begin{abstract}
Anchoring is a cognitive bias connected to the conception of information by every individual. The initial notion created becomes a significant point of reference for all future interpretations of the same object or data, so as to block any further re-elaboration to the initially defined information. The article presents a practical case of a false numerical conception due to anchoring
\end{abstract}

Keywords: anchoring, bias, cognitive, date, conception, morphology

Bias is prejudice in favor of or against one thing, person, or group compared with another, usually in a way considered to be unfair. Biases can be learned implicitly within cultural contexts. People may develop biases toward or against individuals, ethnic groups, sexual or gender identities, nations, religions, social classes, political parties, theoretical paradigms and ideologies within academic domains, or species. "Biased" means one-sided, lacking a neutral viewpoint, or not having an open mind. Bias can come in many forms and is related to prejudice and intuition. 
Cognitive bias involves a deviation from the rational conception of information, which leads to falsely estimating a situation or fact. It is the source and cause of creating an individual reality regarding a particular event or the way one evaluates the data of a given analysis object, which results in a distorted vision inconsistent with objective reality. ${ }^{1}$ It can arise in any individual's common life and behavior, or it might become the reason for a misleading conception of the elements of an analysis object in various scientific approaches. In the case of Forensic Document Examination (FDE), this bias can provoke the so-called confirmation bias, ${ }^{2}$ which could lead to a selective approach to the case data. This could negatively affect the expert's final conclusion, since they only focus on several elements of the case or the investigation object.

One of the cognitive biases is anchoring. Anchoring, ${ }^{3}$ or focalism, is a cognitive bias that describes the tendency for an individual to rely too heavily on an initial piece of information offered (known as the anchor) when making decisions. During decision making, anchoring occurs when individuals use this initial piece of information to make subsequent judgments. Once the value of this anchor is set, all future negotiations, arguments, estimates, etc. are discussed in relation to the anchor. This bias is present when one uses the anchor to interpret future information. For example, the initial price offered for a used car, set either before or at the start of negotiations, sets an arbitrary focal point for all following discussions. Prices discussed during negotiations that are lower than the anchor may seem reasonable, perhaps even cheap to the buyer, even if said prices are still relatively higher than the actual market value of the car. The most important element of this term concerns the focusing

1 M.G. Haselton, D. Nettle, P.W. Andrews, "The evolution of cognitive bias", [in:] The handbook of evolutionary psychology, ed. D.M. Buss, John Wiley \& Sons Inc., Hoboken, NJ 2005, p. 968, https://doi.org/10.1002/9781119125563.evpsych241.

2 M. Merlino, Validity, reliability, accuracy, and bias in forensic signature examination, 2014, p. 11, https://www.ojp.gov/library/publications/validity-reliability-accuracy -and-bias-forensic-signature-identification.

3 F. Kieder et al., "The anchoring bias reflects rational use of cognitive resources", Psychonomic Bulletin and Review 25, 2018, no. 1, pp. 322-349, https://doi.org/10.3758/ s13423-017-1286-8. 
effect (or focusing illusion), which occurs when people place too much importance on one aspect of an event. Anchoring is a psychological heuristic characterized by a difficulty in avoiding its negative effect on one's behavior and durability. According to this heuristic, people start with an implicitly suggested reference point - the anchor - and then make incremental adjustments based on additional information. These adjustments are usually insufficient, giving the initial anchor a great deal of influence over future assessments. Anchoring is connected to several causes. ${ }^{4}$

- Anchoring-and-adjusting: the initial information used as an anchor remains the point of reference, which leads to insufficient subsequent adjustments. For this reason, the final decision remains closer to the anchor.

- Selective accessibility: when given an anchor, the person making a decision is unconsciously looking for the hypothesis closer to the value of the anchor, which is considered the most suitable answer.

- Attitude change: the anchor is indirectly affecting the elaboration of information and biases following judgments towards the answers that are more compatible with it.

Further influencing factors could regard: mood, experience, personality, cognitive ability, and overconfidence.

\section{Practical case}

A negative decision of the court is notified to an attorney of a plaintiff, who has 30 days at their disposal to appeal against it to the Supreme Court. In the document, the court's secretary has to sign the date and time of notification, which is the starting point of the 30-day deadline. This notification takes place on 31.10.2011 and the chronological information is handwritten by filling in the appropriate spaces in the official stamp by the secretary of the court who delivers the notification to the attorney.

4 A. Furnham, H.C. Boo, "A literature review of the anchoring effect", Journal of Socio-Economics 40, 2011, no. 1, p. 37. 

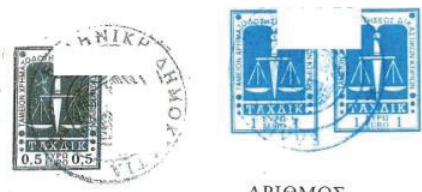

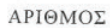

TO

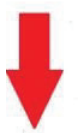

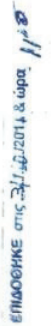



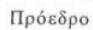

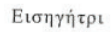

$\kappa \alpha \iota \alpha \pi \delta \quad \Gamma \rho \alpha \mu \mu \alpha \tau \varepsilon \alpha$

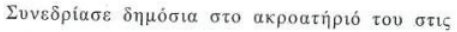

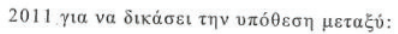

A) TOY EKKA катоіко

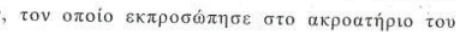

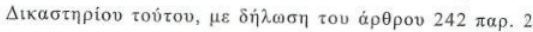

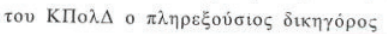

T $\Omega$ N EФE IIB $\Lambda$ HT $\Omega N: 1)$ $\tau \eta v \varepsilon \pi \omega v v \mu i \alpha$

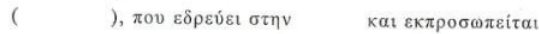

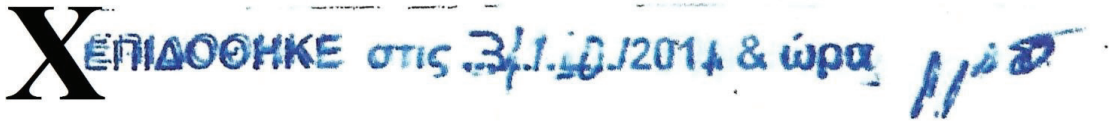

The handwritten information refers to the date (31.10.2011) and the time $(11: 10 \mathrm{am})$ of notification. The attorney, when reading the date which defines the deadline of 30 days, that is " $31 / 10 / 2011$," understands the handwritten date as " $3 / 11 / 2011$." Hence they present the appeal in the court on 2.12.2011 instead of 30.11.2011 and, as a result, the appeal is rejected because of a late submission. The plaintiff sues their attorney, who was to blame for the oversight regarding the date written on the notification. 
We are dealing with a pure case of cognitive bias and, in particular, anchoring. The attorney, after first seeing the written date, conceives the information of " $3 / 11 / 2011$ " and "locks" it in their memory. Before analyzing and explaining why, we have to take a closer look at the particularities of the genuine handwriting of the secretary, who - along with filling in the stamp's empty spaces - has to certify the notification with another official document, which is delivered to the case file:

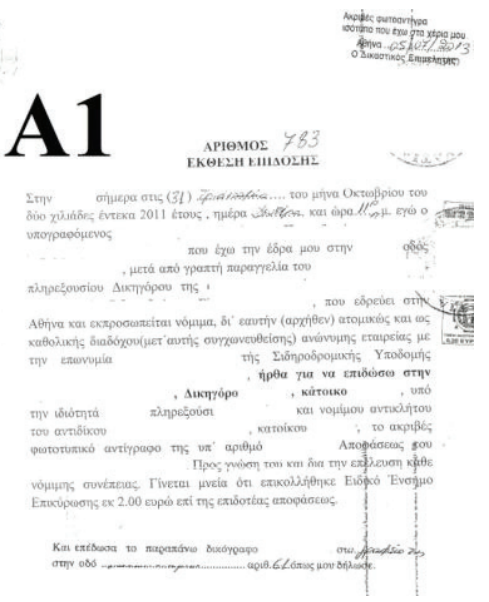

Let us understand and explain why the attorney misunderstood the written date as 3.11.2011 instead of 31.10.2011. Practically, we can read "3/110/2011" (obviously we have the number " 1 " in the center of "110," which is not so clear in the photocopy) in the date, that is day/ month/year. The date is written in the appropriate spaces of the stamp with the handwritten text regarding number 3, the slash (“/”), probably number "110," and the last number " 1 " in the year "2011." In the final part of the stamp, we find the written time: " $11^{10}$." The morphology of the number 1 presents the following variations:

a) simple bar, e.g. "110" (first "1") and "2011" (last "1"),




b) number 1 as bar-horizontal basis, as written in the second "1" of the central number " 110,

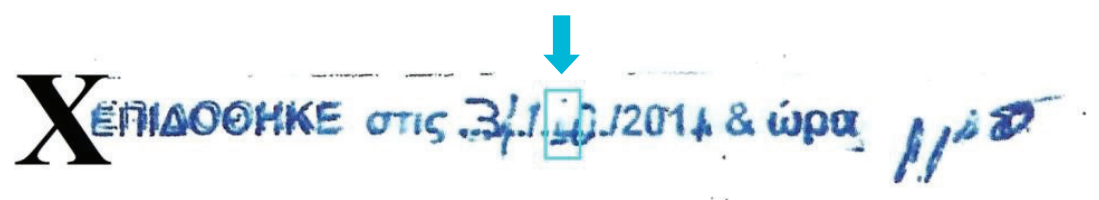

c) double bar, forming a shape similar to a closed "loop," as it appears in the hour of the time "11."

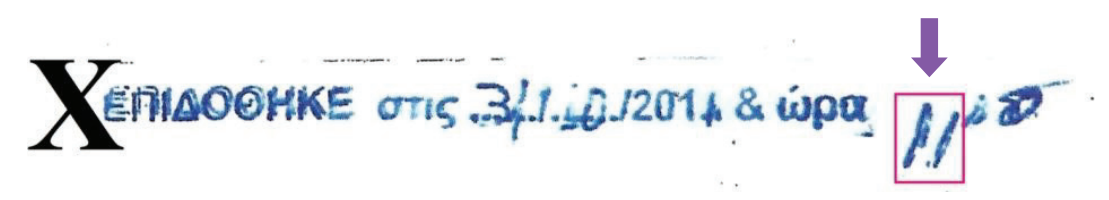

In addition, we have to consider the dimension of the printed characters in the stamp, which subconsciously submits the dimension of the handwritten numbers. To be more specific, the last " 1 " of "2011" is almost exactly of the same dimension as the previous " 1 ," taking into account that the basis of the two specimens is not the same (black lines define the lower and upper endings of the specimens - they are almost parallel).

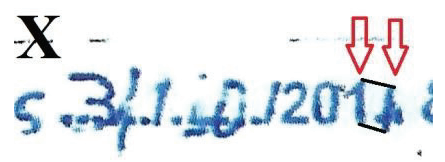

We can also notice that the last number "1" in "2011" has almost the same dimension (about $3 \mathrm{~mm}$ in the copy in our disposal) as the first number " 1 " of " 110 ," which have both the form of a simple bar leaning to the right (and at the same angle).

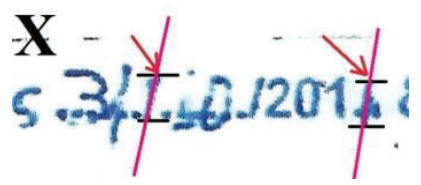

The written time " $11^{10 "}$ indicates the tendency of the hand to write the second number smaller or larger. We have to notice that the time is written in the final part of the stamp in a free space of the paper where 
there is no more subconscious influence of the printed fonts' dimension. This is the reason why the dimension of the numbers in this part is bigger than the ones in the date at the start or in the middle of the stamp.

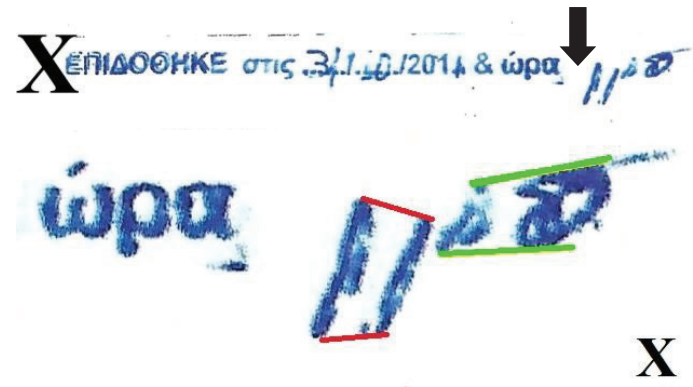

As a result, it is rather logical for the reader to achieve the information of "3/110/2011" without having any information about the intended date, since the reason why we have three numbers in the central part of the month ("110") cannot be explained. So, we do not have a clear notion about the number which defines the month, if it is " 10 " or " 11 ." In any case, the way of forming the numbers does not indicate specifically and legibly "31/10," because the height of the second character (next to " 3 ") is considerably bigger than the first handwritten number 3 , and this fact rather indicates a slash and not number 1, as shown in the analysis above. In addition, it is a common practice to separate the numbers of the date, which define date/month/year, by forming slashes in a much more significant dimension, in order to give a clear indication of the numerical information. This is exactly what seems to have happened in this case, since it is very obvious that the written character next to " 3 " is the biggest of all the handwritten information and much bigger than the first " 3 ," which directly gives the idea of a slash and not number 1 .
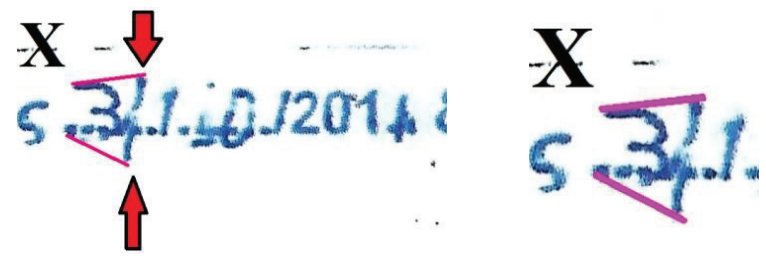
If we consider the second character as number 1 and not a slash, then we can read " $31 / 10 / 2011$ " only if we combine this interpretation with reading the third character (or form) as a stamp slash and not number 1 . So, in order to understand the written date as 31.10.2011, we have to read the second character as 1 and at the same time read the third form as a slash (and not 1). In this case, the hypothetical first slash (third character) presents characteristics analogous to "1" in the stamped "201" of the year - it presents the tendency of forming a "hook" in the upper line of " 1 ," which probably has not been well formed for several reasons (e.g. bad application of the stamp in the paper, bad quality of the copy, bad condition of the stamp due to extended use). On the other hand, the stamped slash before "201" does not present this tendency in the upper part.

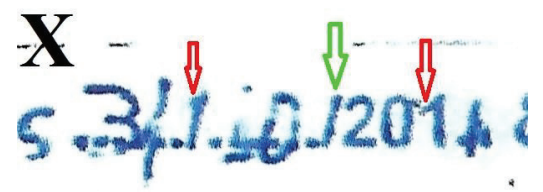

As a result, the written date is not clear and according to the above analysis we can rather read " $3 / 110 / 2011$," which means "3 November 2011," although we still have to explain the reason for the number 0 in the middle (between month and year). However, the justification of the existence of " 0 " in the middle does not allow to clearly, safely, specifically determine the date written.

The interpretation as " 3 November 2011" is confirmed by the characteristics of the number in the certificate of the secretary, which was not delivered to the attorney at first. 


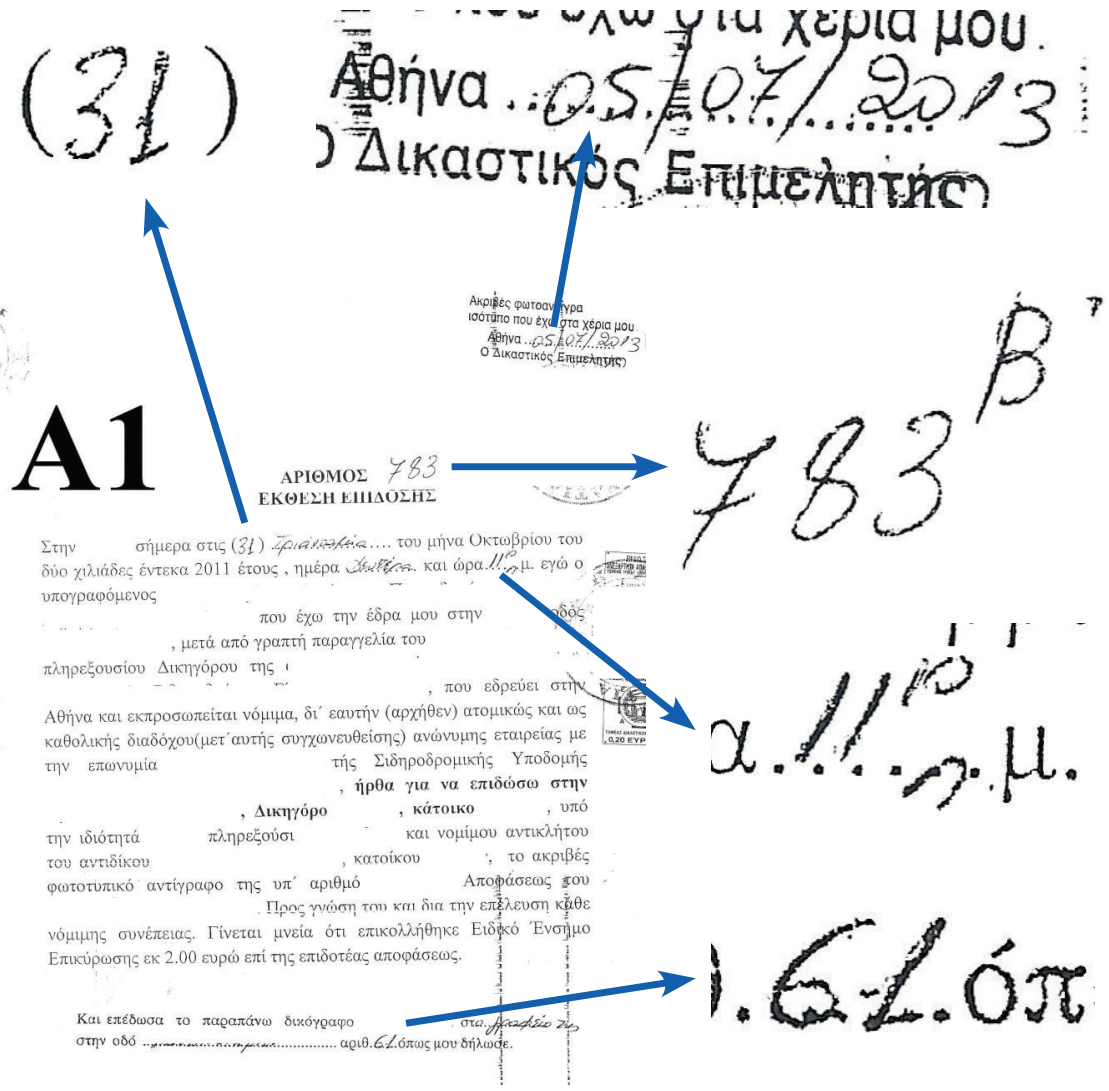

In these various combinations of two numbers, we do not find the second number written in significantly bigger dimensions in comparison to the first. There is always a slight or non-existent difference. This is a stable characteristic of numbers "(31)," "61," and of the time ("11" 10 "). 


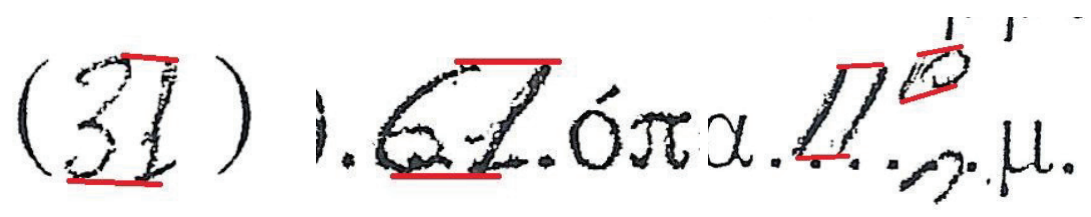

In the case of numbers 31 , where we can have a direct comparison, there is an obvious and undeniable difference between the suspected document and the comparative one in the formation of the dimensions of the numbers.

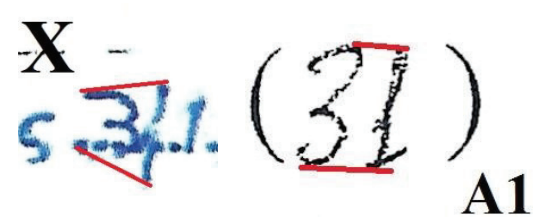

The conception of the date as " 3 November" is further confirmed by the way the date was written by the same secretary in the validation of the document A1 copy. The separation between day/month and month/ year using slashes confirms their graphic habit to clearly differentiate the dimension of the slashes compared to the dimensions of the numbers. The slashes are much bigger than the numbers.

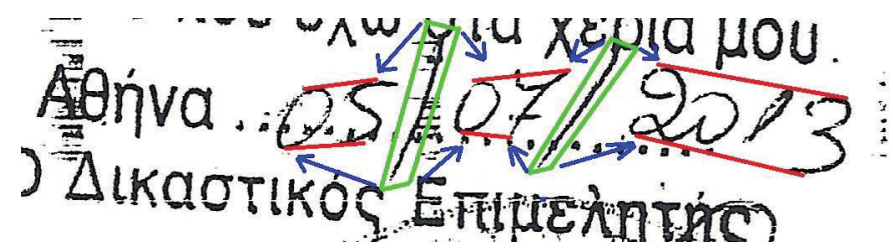

In conclusion, document A1 confirms that the conception of the date in the suspected document, which is not clear and specific, could be rather understood as " $3 / 110 / 2011$," a date which is also not clearly "3 November 2011," but much closer to it. The conception of the date as "31/10/2011" is less probable due to the previously stated reasons. Afterwards, in every attempt by the attorney to re-read the date in the suspected document, they will recall the same date, the "anchor." The eye reads the information starting from the left. For the reader, having understood the second character (after number 3) as a slash means that their mind is "locked" in

Nowa Kodyfikacja Prawa Karnego 59, 2021

(C) for this edition by CNS 
that first impact as " $3 /$. " Then, they achieve the information of the month in the first two " 1 "s as " 11 " and, although they cannot logically explain the presence of the following number " 0 ," they do not attempt to resolve the problem, because they have already completed the information needed, that is " $3 / 11$." The conception of the year is not important, since we are all aware of the current year, and the main information needed here is the starting date of the period of 30 days. So, the individual tends to rely too heavily on an initial piece of information (the "anchor") when making further decisions, a psychological process called anchoring or focalism. Every time the individual tries to read the information again, due to anchoring the initial piece of information is recalled in order to make all subsequent judgments. Once the value of this anchor is set, all future negotiations, arguments, estimates, etc. are discussed or applied in relation to the anchor. This means that every time the attorney thinks or tries to re-read the date in the notified document they will read "3/11." In addition to this, the anchor will define their behavior (in this case: the date of presenting the appeal to the court).

The effect of anchoring is very important in the cases of forgery due to the fact that the forger may misunderstand several parts of the model to imitate (handwriting, signature, or squiggle). We typically expect a morphological difference in a suspected specimen to be the result of the writer's genuine graphic variability. This might be a misleading hypothesis which could distort our conclusion, especially if we take into consideration the potential effect of anchoring.

\section{References}

Haselton M.G., Nettle D., Andrews P.W., "The evolution of cognitive bias", [in:] The handbook of evolutionary psychology, eds. D.M. Buss, Hoboken, NJ 2005, https://doi. org/10.1002/9781119125563.evpsych241.

Furnham A., Boo H.C., "A literature review of the anchoring effect", Journal of SocioEconomics 40, 2011, no. 1.

Merlino M., Validity, reliability, accuracy, and bias in forensic signature examination, 2014, p. 11, https://www.ojp.gov/library/publications/validity-reliability-accuracyand-bias-forensic-signature-identification.

Kieder F. et al., "The anchoring bias reflects rational use of cognitive resources", Psychonomic Bulletin and Review 25, 2018, no. 1, pp. 322-349, https://doi.org/10.3758/ s13423-017-1286-8. 